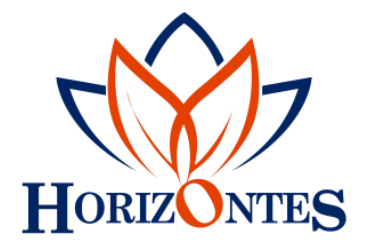

Horizontes. Revista de Investigación en Ciencias de la Educación Https://doi.org/10.33996/revistahorizontes.v5i18.187

Abril - junio 2021

Volumen 5 / No. 18

ISSN: $2616-7964$

ISSN-L: $2616-7964$

www.revistahorizontes.org

pp. $447-461$

\title{
Valores interpersonales y pensamiento crítico en estudiantes de una universidad de Lima, 2020
}

\author{
Interpersonal values and critical thinking in students of a university in Lima, \\ 2020
}

Valores interpessoais e pensamento crítico em alunos de uma universidade em Lima, 2020

ARTÍCULO DE INVESTIGACIÓN

$\begin{aligned} & \text { Jorge Alberto Flores Morales } \\ & \text { 1 } \\ & \text { jorgefloresm@unife.pe }\end{aligned}$
https://orcid.org/0000-0002-3678-5511

\author{
Lidia Neyra Huamani ${ }^{2}$ \\ c18727@utp.edu.pe \\ https://orcid.org/0000-0001-6261-2190
}
1Universidad Femenina del Sagrado Corazón, Lima-Perú ${ }^{2}$ Universidad Tecnológica del Perú, Lima-Perú

Recibido 08 de enero 2021 | Arbitrado y aceptado 15 de febrero 2021 | Publicado en 01 abril 2021

\section{RESUMEN}

El objetivo del presente estudio fue determinar la relación entre el Pensamiento crítico y los Valores interpersonales en estudiantes universitarios del I ciclo de una universidad privada de Lima. La muestra fue intencional conformada por 115 participantes. El diseño es no experimental correlacional de enfoque cuantitativo, los datos se analizaron a través de la estadística no paramétrica de Spearman. Se aplicó los cuestionarios de Pensamiento crítico (Zaldivar, 2010) y el cuestionario de Valores Interpersonales (SIV). Los resultados arrojaron que existe una correlación positiva y de mediana intensidad (rho = ,513), por lo cual, la direccionalidad de la correlación es correcta, permitiendo aducir que puntajes altos en los valores interpersonales están relacionados con los puntajes altos del pensamiento crítico: A mayores valores interpersonales, mayor pensamiento crítico.

Palabras clave: Competencia; educación superior; estudiantes; pensamiento crítico, valores

\section{ABSTRACT}

The objective of this study was to determine the relationship between Critical Thinking and Interpersonal Values in university students from the 1 st cycle of a private university in Lima. The sample was intentional made up of 115 participants. The non-experimental correlational design with a quantitative approach, the data were analyzed through Spearman's non-parametric statistics. The Critical Thinking questionnaires (Zaldivar, 2010) and the Interpersonal Values questionnaire (SIV) were applied. The results showed that there is a positive and medium-intensity correlation (rho $=.513$ ), therefore, the directionality of the correlation is correct, allowing to argue that high scores in interpersonal values are related to high scores for critical thinking: The higher the interpersonal values, the more critical thinking.

Key words: Competence; higher education; students; critical thinking, values

\section{RESUMO}

A gestão de projetos na construção civil e a análise de casos reais ganharam importância nas últimas décadas. 0 objetivo do estudo é analisar retrospectivamente a gestão de um projeto de construção de infraestrutura de um teleférico, evidenciando os problemas e soluções adotados durante sua execução. Aplicando o método de estudo de caso (Línea Blanca e Celeste do Teleférico de La Paz, Bolívia), a gestão do projeto foi investigada e avaliada, seguindo 4 etapas: descrição do projeto, identificação de problemas, análise posterior, descrição das soluções implementadas. Essa metodologia permitiu a identificação de 5 problemas transcendentais, relacionados a: modificação da funcionalidade de operação, liberação de propriedades, mudanças de layout e modificação da infraestrutura e instabilidade de taludes. Conclui-se que diante de problemas de grande magnitude, a gestão do projeto foi fundamental. Eles também destacam as lições aprendidas que são muito úteis para projetos semelhantes em todo o mundo.

Palavras-Chave: Teleférico; transporte por cabo; gerenciamento de projetos; contrução civil 


\section{INTRODUCCIÓN}

La calidad del pensamiento crítico estará sujeto a la profundidad de los valores interiorizados por la persona. La experiencia valorativa que el sujeto haya practicado a través del tiempo será el insumo no solo de su comportamiento sino también de un juicio ponderado cercano lo más posible a la realidad y no por intereses particulares. Esta experiencia valorativa se va formando desde la infancia con la ayuda de los padres en un primer momento y luego de los maestros (Dicado, Campi y Saa, 2019). Pero donde realmente se consolida esta relación de valores y pensamiento crítico es en el ámbito universitario es allí donde se toman decisiones y opciones de comportamiento sin el tutelaje de padres y maestros.

La toma de decisiones basadas en valores va a fortalecer el desarrollo del pensamiento crítico organizándolo dentro de los parámetros de la verdad, realidad y sentido. La persona que ha sido formado en valores pondrá en práctica estos a la hora de juzgar la realidad a través de la reflexión del entorno. No se dejará llevar por intereses subalternos ni por ideas mezquinas, juzgará los diversos puntos de vista y también analizará las evidencias expuestas para luego contrastarla bajo criterios objetivos y que estas sirvan para dar una mejor solución a los problemas distinguiendo lo importante de lo urgente.

En un ambiente de incertidumbre, propiciada por la pandemia del Covid-29, los acontecimientos aparecen de manera inusitada como las nuevas variantes del virus, cuarentenas impuestas, inestabilidad laboral, riesgo de muerte, etc. Estas situaciones nos congojan y crean una serie de preocupaciones con poco tiempo para reflexionar críticamente y elaborar mejores respuestas ante estos hechos luctuosos. Todo ello, ha generado en muchas personas vacíos existenciales que al no encontrar refugio en los valores en especial en los interpersonales terminan sucumbiendo ante los antivalores como la desesperanza, frustración, ansiedad, miedo, etc.

La pandemia ha puesto en jaque no solo la dimensión de la salud y el aprecio al valor de la vida sino también todos aquellos elementos que nos daban aparente seguridad y nos hacía sentir parte del engranaje económico-social. Entonces para muchas personas lo que les definía no era su conciencia de ser personal y ser en relación sino su activismo expresado en el trabajo, la reunión con amigos, ir de compras, las celebraciones familiares, los viajes y paseos, entre otros, o siguen el aforismo cartesiano "pienso luego, existo", sino el "compro, luego existo", "trabajo, luego existo", "Salgo a fiesta, luego existo", "tengo sexo, luego existo", entre otros.

Ahora que se vive la incertidumbre producida por el covid-19 por sus efectos fatales ha llevado a sopesar lo relativo de la existencia humana. Ante ella tenemos dos respuestas la que nace de un pensamiento crítico del entorno con fuerte presencia axiológica que verá motivos para dar siempre lo mejor de sí y otros que solo vislumbran tragedia con la consecuencia de la perdida de sentido.

La formación en valores se nutre desde el hogar y se va consolidando en el tiempo, la experiencia escolar y universitaria es el espació de relación con el otro donde ejerzo de manera no tutelar mis convicciones personales y doy razón o no de mi proceder. El ser formado en valores será el medio que guie el pensamiento juicioso sobre la realidad y la postura frente a la verdad o el engaño.

El termino valor procede etimológicamente del griego "Axio" referido a lo digno o que tiene valor. La axiología es el 
estudio de los valores y en la cual la conducta humana ajusta o no su comportamiento. Entonces el actuar axiológico tiene implicancias no solo personales sino interpersonales.

Frondizi (1990) definirá los valores como aquellas cualidades fundamentales que ante las propiedades del objeto el sujeto reacciona, estas cualidades serán fundamentales o sensibles. Tenemos también el acercamiento del estudio de los valores por Rokeach (1973) que concibe el valor como una creencia que permanece en el tiempo generando un modo especifico de conducta socialmente preferible. Además, clasifica los valores en dos grandes grupos: Valores Instrumentales (Valores morales y valores de suficiencia) y Valores Terminales (personales, sociales y culturales).

Los valores han de entenderse como la adopción que el ser humano realiza en la actualidad que le permiten actuar con seguridad y trascender en el tiempo, según la propuesta de Casares, González y Siliceo, (1999).

El autor del Cuestionario de Valores Interpersonales (Gordon Allpor, 1977) entiende estos valores como aquellos que ayudan en las relaciones del individuo con sus semejantes sirviendo de ajuste a nivel familiar, social, laboral, etc. Cuando no se da estos ajustes el comportamiento y la actitud social cambia expresados en conductas delictivas o antisociales desde la adolescencia. (GázquezLinares, Pérez-Fuentes, Carrión-Martínez y Molero, 2015; Pérez, Linares, Molero, Soler y Martín, 2015).

Al plantear el termino valores interpersonales refiere aquella disposición o preferencia que tenemos hacia los otros poniendo como medio de relación un conjunto de valores que consideramos fundamentales para el buen entendimiento. Estos valores que ponemos en juego al relacionarnos con los demás parten de un proceso reflexionado y critico que consideramos esencial en la convivencia humana, por ejemplo, el tratar al "otro" con respeto lo hago porque considero que no debe haber ningún tipo de discriminación entre las personas y ello trae bienestar social.

Es en el campo educativo donde se empieza afianzar las relaciones interpersonales constructivas, es en el grupo de amigos donde se van creando amistades duraderas y también vivencias con intensidad otros valores como el amor, empatía, honestidad, la solidaridad etc. (Kleptsova, et al., 2018).

El pensar críticamente no es uso exclusivo de filósofos, sociólogos o políticos. El pensar críticamente es una competencia de toda persona que no acepta ideas o acontecimientos sin previo análisis e independientemente de donde provenga; implica rechazar todo pensamiento dogmático o empirista que no es capaz de aceptar otras posibilidades de la realidad. El pensador crítico es un enamorado de los argumentos, del estudio e investigación que busca evidenciar los hechos lejos de sofismas o ideas escépticas. Es agregado también que el pensamiento crítico implica el:

Proceso de analizar y evaluar el pensamiento con el propósito de mejorarlo. El pensamiento crítico presupone el conocimiento de las estructuras más básicas del pensamiento (los elementos del pensamiento) y los estándares intelectuales más básicos del pensamiento (estándares intelectuales universales). La clave para desencadenar el lado creativo del pensamiento crítico (la verdadera mejora del pensamiento) está en reestructurar el pensamiento como resultado de analizarlo y evaluarlo de manera efectiva. (Richard y Elder, 2005, p. 7). 
Entonces el pensar críticamente no puede ser reducido a una serie de características o habilidades cognitivas, sino que resulta ser una manera de actuar y ser en el mundo. Pero el ser un pensador critico no está exento de equivocarse o realizar una mala inferencia ya que nuestro actuar no es a manera de un sistema informático y puedo actuar alejándome de los valores o ir en contra de ellos promoviendo un antivalor. Es por eso que se afirma que el pensamiento crítico es la "disposición que adopta un sujeto a la hora de interpretar y evaluar la información que dispone." (Zaldivar, 2010, p. 10). Ella lleva al sujeto a reconocer supuestos que nace producto de un análisis serio, a interpretar la realidad de manera equilibrada y evaluar los argumentos consistentes de los débiles. Así también Facione (2007 p. 8) al presentar lo que considera pensamiento crítico lo señala como una "forma de juicio serio o de toma de decisión reflexiva, en sentido muy real el pensamiento crítico impregna todo." Ello ligado a la forma de cómo se plantea la pregunta, el estudio se enfoca en los acontecimientos, las definiciones de los problemas específicos, entre otros, donde se generan habilidades de interpretación, análisis, evaluación, inferencias, explicación y autorregulación.

El ejercicio de la experiencia valorativa asumida a través de un pensamiento reflexivo y crítico ayudará al estudiante hacer frente a la presión social que se expresa a través del llamado "efecto bandwagon" o "efecto de arrastre", en el cual muchas personas se dejan llevar "por lo que todo el mundo hace o dice", apartando de sí la capacidad reflexiva previa que implica el investigar, analizar y tomar una decisión.

Este "efecto Bandwangon" es utilizado por muchos actores sociales o del espectáculo que tratan de imponer modas, ideologías, nuevos hábitos de consumo, etc. que se considera todo ello parte del negocio o propaganda. También, están los "influencers" que llegan a los jóvenes estudiantes de educación básica regular y universitarios de manera específica, que son los futuros consumidores y seguidores de estas nuevas tendencias que ingresan a sus vidas a través del impacto mediáticos de las redes sociales.

Todo lo señalado hasta el momento forman parte de una dinámica socio cultural que existe y existirán a lo largo del tiempo y con nuevas formas de interacción. Lo importante es, que la postura de vida que se vaya a optar nazca de una decisión libre, que busque los detalles y matices de aquello que se ofrece, con ayuda del pensamiento crítico, creativo, de resolución de problemas y de vivencia axiológica.

El pensar cítricamente es una herramienta potentísima en la captación de valores en su cuestionamiento, en su puesta en práctica y la asunción de ella como parte de mi existencia personal, pero también es responsabilidad de los centros de estudios escolares y en especial de los universitarios que han de favorecer planes de trabajo que promuevan valores éticos y morales no solo a nivel interpersonal sino también de responsabilidad social.

La educación universitaria a través de su sistema de enseñanza y aprendizaje pretende no solo trasmitir su experiencia $y$ conocimientos para tener al final de proceso un buen profesional íntegro con valores y que sepa responder con altura frente a los sistemas de corrupción que pueda darse en cualquier ámbito de su labor profesional.

Hay que estar atentos a otro fenómeno social, como son los "Fake news" donde los argumentos falaces desean aparecer como verdades científicas o altamente "creíbles". 
Ante ello, la mejor forma de contrarrestar estas informaciones es el desarrollar el pensamiento crítico que ayude a identificar inconsistencias del mensaje, desarrollar la conexión de ideas a través del análisis de estas, de abordar las premisas de manera sistemática, para así llegar a emitir juicios finales lo más cercano a la realidad con argumentos sólidos y no emotivos.

El actuar o tomar una postura acrítica sobre los hechos, pareciera ser que es de personas menos instruidas que pueden ser fácilmente influenciadas. Pero la realidad demuestra que no es así, muchos dictadores subieron al poder, no solo por el gran caudal de votos que recibieron en primera instancia, sino que hombres provenientes de círculos intelectuales los apoyaron y tomaron partido por ellos, dada su imagen de confianza proyectaban a su público elector y con el compromiso de usar la mano dura contra los problemas que aquejaban al país, pero no se hizo el análisis crítico su propuesta política e ideológica y si representaba los verdaderos valores del ciudadano libre.

El sentido crítico acompañado de una formación axiológica ayuda a distinguir lo real de lo ficticio, de la ciencia de la que no la es, del oportunismo individualista al bien común, entre otros. Esto ha de ser también, una tarea del estudiante de educación superior, ya que las aulas universitarias no son solo de acopio de conocimientos, sino también de debate, reflexión, de ejercicio de nuestra libertad en el sano respeto al otro, donde se debaten ideas de todo tipo con argumentos sólidos y no de "Argumento ad hominem"

\section{MÉTODO}

El presente estudio parte del enfoque cuantitativo de con diseño no experimental, transeccional y correlacional. El instrumento fue el cuestionario de Valores Interpersonales (SIV) de Gordon Alport que mide los valores que el sujeto adopta e inciden en la relación con los demás y estos son Soporte, Conformidad, Reconocimiento, Independencia, Benevolencia y Liderazgo. El otro instrumento de aplicación fue el cuestionario de Pensamiento crítico de Zaldívar (2010). El cual, consta de 20 reactivos y tres dimensiones que son: Reconocimiento de asunciones, Interpretación y Evaluación de argumentos basado en el trabajo de Watson y Gleser (1980). La muestra de estudio estuvo conformada por 115 estudiantes del I ciclo de una universidad particular de Lima.

\section{RESULTADOS}

\section{Resultados descriptivos}

A continuación se muestran los resultados basados en los valores interpersonales que han sido en gran proporción de nivel alto en la muestra de estudio ( $>60 \%$ ), existiendo menor nivel de prevalencia de estos valores interpersonales en la muestra seleccionada para el estudio. (Tabla 1 ). 
Tabla 1. Valores interpersonales en la muestra de estudio.

\begin{tabular}{cccccc}
\hline & & Frecuencia & Porcentaje & Porcentaje válido & Porcentaje acumulado \\
\hline \multirow{4}{*}{ Válido } & Bajo & 7 & 6,1 & 6,1 & 6,1 \\
& Medio & 30 & 26,1 & 26,1 & 32,2 \\
& Alto & 78 & 67,8 & 67,8 & 100,0 \\
& Total & 115 & 100,0 & 100,0 & \\
\hline
\end{tabular}

Fuente: Base de datos de la investigación.

En cuanto a la Tabla 2, se puede describir mayor prevalencia de nivel promedio en el pensamiento crítico en más de la mitad de los estudiantes evaluados $(53,9 \%)$. El menor valor encontrado se centró en más del $6 \%$ de sujetos que presentaron bajo nivel del pensamiento crítico.

Tabla 2. Pensamiento crítico en la muestra de estudio.

\begin{tabular}{cccccc}
\hline & & Frecuencia & Porcentaje & Porcentaje válido & Porcentaje acumulado \\
\hline \multirow{4}{*}{ Válido } & Bajo & 7 & 6,1 & 6,1 & 6,1 \\
& Promedio & 62 & 53,9 & 53,9 & 60,0 \\
& Alto & 46 & 40,0 & 40,0 & 100,0 \\
& Total & 115 & 100,0 & 100,0 & \\
\hline
\end{tabular}

Fuente: Base de datos de la investigación.

\section{Análisis de normalidad de datos}

Debido a que los datos obtenidos superan la cantidad muestral de 30 unidades, previamente se consideró aplicar la prueba de análisis de normalidad Kolmogorov-Smirnov (K-S 1), para calcular el criterio de normalidad en la distribución de los datos obtenidos.

Tabla 3. Datos de normalidad en variables valores interpersonales y pensamiento crítico y análisis de dimensiones

\begin{tabular}{|c|c|c|c|c|c|c|c|c|c|}
\hline & & VAR_PC & VAR_VINT & D1_SOP & D2_CONF & D3_REC & D4_IND & D5_BENEV & D6_LID \\
\hline & & 115 & 115 & 115 & 115 & 115 & 115 & 115 & 115 \\
\hline Parámetros & Media & 81,37 & 97,62 & 38,57 & 42,97 & 45,01 & 36,41 & 39,42 & 42,05 \\
\hline & $\begin{array}{l}\text { Desviación } \\
\text { estándar }\end{array}$ & 21,772 & 52,104 & 20,315 & 20,791 & 22,214 & 16,253 & 14,623 & 14,747 \\
\hline Máximas & Absoluta & 092 & 174 & 182 & 188 & 123 & 180 & 196 & 163 \\
\hline $\begin{array}{l}\text { diferencias } \\
\text { extremas }\end{array}$ & Positivo & ,080 & 174 & 182 & 188 & 114 & 180 & 196 & 163 \\
\hline & Negativo &,- 092 &,- 101 &,- 120 &,- 110 &,- 123 &,- 122 &,- 102 &,- 102 \\
\hline Estadístico c & e prueba & 092 & ,174 & 182 & 188 & 123 & 180 & 196 & 163 \\
\hline Sig. asintótic & a (bilateral) &, $017 \mathrm{c}$ &, $000^{c}$ &, $000^{c}$ &, $000^{c}$ &, $000^{c}$ &, $000^{c}$ &, $000^{c}$ &, $000^{c}$ \\
\hline
\end{tabular}

Fuente: Base de datos de la investigación. 
De acuerdo con la Tabla 3, se puede observar que los índices de significancia son menores a 0,05 en todas las dimensiones como en las variables. Por cuanto, se decidió por aplicar pruebas estadísticas no paramétricas en el análisis de todas las correlaciones (prueba de Spearman). Para el análisis correlacional, se consideró como p-valor o valor de contraste al 5 $\%$ del total de probabilidad. Es decir 0,005 para todos los contrastes desarrollados en lo posterior del análisis.

\section{Resultados inferenciales}

Contraste de hipótesis general: valores interpersonales y pensamiento crítico.

$$
\begin{aligned}
\mathrm{Hi}= & \text { A mayores valores interpersonales, } \\
& \text { mayor pensamiento crítico. } \\
\mathrm{Ho}= & \text { Los valores interpersonales y } \\
& \begin{array}{l}
\text { pensamiento } \\
\text { correlacionan. }
\end{array}
\end{aligned}
$$

Tabla 4. Análisis de correlación entre los valores interpersonales y el pensamiento crítico.

\begin{tabular}{ccccc}
\hline & & $\begin{array}{c}\text { Pensamiento } \\
\text { crítico }\end{array}$ & $\begin{array}{c}\text { Valores } \\
\text { interpersonales }\end{array}$ \\
\hline & $\begin{array}{c}\text { Pensamiento } \\
\text { crítico }\end{array}$ & Coeficiente de correlación & 1,000 &, $513^{* *}$ \\
Rho de & Sig. (bilateral) & &, 000 \\
Spearman & $\begin{array}{c}\text { Valores } \\
\text { interpersonales }\end{array}$ & Coeficiente de correlación &, $513^{* *}$ & 115 \\
& & Sig. (bilateral) &, 000 & 1,000 \\
& & $\mathrm{~N}$ & 115 & 115 \\
\hline
\end{tabular}

Fuente: Base de datos de la investigación.

La Tabla 4 presenta la correlación entre los valores interpersonales y el pensamiento crítico, siendo esta de tipo significativa con índices menores al límite de significancia establecido ( $p<005)$, por cuanto, las evidencias permiten aceptar la existencia de relación. Por otro lado, está correlación es positiva y de mediana intensidad $(r h o=, 513)$, por lo cual, la direccionalidad de la correlación es correcta, permitiendo aducir que puntajes altos en los valores interpersonales están relacionados con los puntajes altos del pensamiento crítico: A mayores valores interpersonales, mayor pensamiento crítico.

\section{Contraste de hipótesis específicas}

\section{Soporte y pensamiento crítico}

$\mathrm{Hi}=\mathrm{A}$ mayor valor interpersonal de soporte mayor pensamiento crítico.

$\mathrm{Ho}=\mathrm{El}$ valor interpersonal de soporte $\mathrm{y}$ el pensamiento crítico no se correlacionan. 
Tabla 5. Análisis de correlación entre el valor de soporte y el pensamiento crítico.

\begin{tabular}{|c|c|c|c|c|}
\hline & & & Pensamiento crítico & Soporte \\
\hline \multirow{6}{*}{$\begin{array}{l}\text { Rho de } \\
\text { Spearman }\end{array}$} & \multirow{3}{*}{$\begin{array}{l}\text { Pensamiento } \\
\text { crítico }\end{array}$} & Coeficiente de correlación & 1,000 &, $249^{* *}$ \\
\hline & & Sig. (bilateral) & & 007 \\
\hline & & $\mathrm{N}$ & 115 & 115 \\
\hline & \multirow[t]{3}{*}{ Soporte } & Coeficiente de correlación &, $249^{* *}$ & 1,000 \\
\hline & & Sig. (bilateral) & ,007 & \\
\hline & & $\mathrm{N}$ & 115 & 115 \\
\hline
\end{tabular}

Fuente: Base de datos de la investigación.

La Tabla 5, demuestra la existencia de relación respecto al índice p-valor inicial. De esto se puede asumir inicialmente que, la correlación es significativa $(p<.005)$, a su vez, en el análisis se puede observar que este índice de correlación de baja intensidad (rho $=$,249), pero es de tipo positiva. De estas evidencias se puede colegir que existen valores altos en ambas variables, estando correlacionados entre sí, ante esto se puede aceptan la hipótesis alterna como verdadera:
A mayor valor interpersonal de soporte mayor pensamiento crítico.

\section{Conformidad y pensamiento crítico}

$\mathrm{Hi}=\mathrm{A}$ mayor valor interpersonal de conformidad mayor pensamiento crítico.

$\mathrm{Ho}=\mathrm{El}$ valor interpersonal de conformidad $\mathrm{y}$ el pensamiento crítico no se correlacionan.

Tabla 6. Análisis de correlación entre el valor de conformidad y el pensamiento crítico.

\begin{tabular}{|c|c|c|c|c|}
\hline & & & Pensamiento crítico & Conformidad \\
\hline \multirow{6}{*}{$\begin{array}{c}\text { Rho de } \\
\text { Spearman }\end{array}$} & \multirow{3}{*}{$\begin{array}{l}\text { Pensamiento } \\
\text { crítico }\end{array}$} & Coeficiente de correlación & 1,000 &, $316^{* *}$ \\
\hline & & Sig. (bilateral) & & 001 \\
\hline & & $\mathrm{N}$ & 115 & 115 \\
\hline & \multirow[t]{3}{*}{ Conformidad } & Coeficiente de correlación &, $316^{* *}$ & 1,000 \\
\hline & & Sig. (bilateral) & , 001 & \\
\hline & & $\mathrm{N}$ & 115 & 115 \\
\hline
\end{tabular}

Fuente: Base de datos de la investigación. 
En relación con la Tabla 6, se puede aseverar la existencia de relación entre las variables pensamiento crítico y el valor interpersonal de conformidad, siendo significativa $(p$-valor $=, 001 ; p<.005)$. La intensidad de correlación es de moderada intensidad y de tipo positiva ( $r h o=, 316)$, por lo que se puede determinar que la hipótesis alterna cuenta con evidencias para aceptarla: A mayor valor interpersonal de conformidad mayor pensamiento crítico.

\section{Reconocimiento y pensamiento crítico}

$\mathrm{Hi}=\mathrm{A}$ mayor valor interpersonal de reconocimiento mayor pensamiento crítico.

$\mathrm{Ho}=\mathrm{El} \quad$ valor interpersonal de reconocimiento y el pensamiento crítico no se correlacionan.

Tabla 7. Análisis de correlación entre el valor de reconocimiento y el pensamiento crítico.

\begin{tabular}{ccccc}
\hline & & $\begin{array}{c}\text { Pensamiento } \\
\text { crítico }\end{array}$ & Reconocimiento \\
\hline & $\begin{array}{c}\text { Pensamiento } \\
\text { crítico }\end{array}$ & Coeficiente de correlación & 1,000 &, $308^{* *}$ \\
& & Sig. (bilateral) & &, 001 \\
$\begin{array}{c}\text { Rho de } \\
\text { Spearman }\end{array}$ & Reconocimiento & N & 115 & 115 \\
& & Coeficiente de correlación &, $308^{* *}$ & 1,000 \\
& & Sig. (bilateral) &, 001 & \\
& & $\mathrm{~N}$ & 115 & 115 \\
\hline
\end{tabular}

Fuente: Base de datos de la investigación.

En cuanto a la Tabla 7, el análisis correlacional entre la variable pensamiento crítico y el reconocimiento como un valor interpersonal ha presentado significancia relativa al índice p-valor (sig. $=, 001$ ), por cuanto podemos aceptar la existencia de la relación entre ambas. También se ha encontrado que el índice de Spearman es positivo $\mathrm{y}$ de moderada intensidad ( $r h o=$ ,308), por lo cual se acepta que la hipótesis alterna es cierta: A mayor valor interpersonal de reconocimiento mayor pensamiento crítico.

\section{Independencia y pensamiento crítico}

$\mathrm{Hi}=\mathrm{A}$ mayor valor interpersonal de independencia mayor pensamiento crítico.

Ho $=$ El valor interpersonal de independencia $y$ el pensamiento crítico no se correlacionan. 
Tabla 8. Análisis de correlación entre el valor de independencia y el pensamiento crítico.

\begin{tabular}{ccccc}
\hline & & $\begin{array}{c}\text { Pensamiento } \\
\text { crítico }\end{array}$ & Independencia \\
\hline & $\begin{array}{c}\text { Pensamiento } \\
\text { crítico }\end{array}$ & Coeficiente de correlación & 1,000 &, $374^{* *}$ \\
& & Sig. (bilateral) & &, 000 \\
$\begin{array}{c}\text { Rho de } \\
\text { Spearman }\end{array}$ & Independencia & Coeficiente de correlación & 115 & 115 \\
& & Sig. (bilateral) &, 000 & 1,000 \\
& & $\mathrm{~N}$ & 115 & 115 \\
\hline
\end{tabular}

Fuente: Base de datos de la investigación.

En relación a la Tabla 8 , el valor de correlación obtenido demuestra que la correlación entre el valor de independencia y el pensamiento crítico es significativa $(p<$. 005), por lo que se acepta la existencia de relación de ambas variables. De igual modo, la intensidad de relación es de tipo moderada $(r h o=, 374)$. Sin embargo, la positividad de esta relación permite contar con las evidencias necesarias como para determinar que la hipótesis alterna debe gozar de aceptación estadística: A mayor valor interpersonal de independencia mayor pensamiento crítico.

\section{Benevolencia y pensamiento crítico}

$\mathrm{Hi}=\mathrm{A}$ mayor valor interpersonal de benevolencia mayor pensamiento crítico.

$\mathrm{Ho}=\mathrm{El}$ valor interpersonal de benevolencia y el pensamiento crítico no se correlacionan.

Tabla 9. Análisis de correlación entre el valor de benevolencia y el pensamiento crítico.

\begin{tabular}{|c|c|c|c|c|}
\hline & & & Pensamiento crítico & Benevolencia \\
\hline \multirow{6}{*}{$\begin{array}{c}\text { Rho de } \\
\text { Spearman }\end{array}$} & \multirow{3}{*}{$\begin{array}{l}\text { Pensamiento } \\
\text { crítico }\end{array}$} & Coeficiente de correlación & 1,000 &, $310^{* *}$ \\
\hline & & Sig. (bilateral) & & 001 \\
\hline & & $\mathrm{N}$ & 115 & 115 \\
\hline & \multirow[t]{3}{*}{ Benevolencia } & Coeficiente de correlación &, $310^{* *}$ & 1,000 \\
\hline & & Sig. (bilateral) & 001 & \\
\hline & & $\mathrm{N}$ & 115 & 115 \\
\hline
\end{tabular}

Fuente: Base de datos de la investigación. 
En cuanto a la Tabla 9, sobre el análisis de correlación entre el valor de benevolencia y el pensamiento crítico permitió obtener datos que sustentan la relación entre ambas variables, siendo esta de tipo significativa (sig. $=, 001 ; \mathrm{p}<.005)$. A su vez, la correlación reportada permite aducir que dicha relación presenta intensidad moderada y de tipo positiva (rho $=, 310$ ), por lo que se acepta la hipótesis alterna como respuesta a este contraste: A mayor valor interpersonal de benevolencia mayor pensamiento crítico.

\section{Liderazgo y pensamiento crítico}

$\mathrm{Hi}=\mathrm{A}$ mayor valor interpersonal de liderazgo mayor pensamiento crítico.

$\mathrm{Ho}=\mathrm{El}$ valor interpersonal de liderazgo y el pensamiento crítico no se correlacionan.

Tabla 10. Análisis de correlación entre el valor de liderazgo y el pensamiento crítico.

\begin{tabular}{|c|c|c|c|c|}
\hline & & & Pensamiento crítico & Liderazgo \\
\hline \multirow{6}{*}{$\begin{array}{l}\text { Rho de } \\
\text { Spearman }\end{array}$} & \multirow{3}{*}{$\begin{array}{l}\text { Pensamiento } \\
\text { crítico }\end{array}$} & Coeficiente de correlación & 1,000 &, $281^{* *}$ \\
\hline & & Sig. (bilateral) & & ,002 \\
\hline & & $\mathrm{N}$ & 115 & 115 \\
\hline & \multirow[t]{3}{*}{ Liderazgo } & Coeficiente de correlación &, $281^{* *}$ & 1,000 \\
\hline & & Sig. (bilateral) & ,002 & \\
\hline & & $\mathrm{N}$ & 115 & 115 \\
\hline
\end{tabular}

Fuente: Base de datos de la investigación.

La Tabla 10, expresa el índice de significancia de la correlación entre las variables pensamiento crítico y el valor interpersonal de liderazgo $(p<.005)$, por lo que se aduce que ambas se encuentran relacionadas. El índice de intensidad de esta relación (rho $=, 281$ ), apoya el argumento de que esta relación es de baja intensidad, pero a su vez positiva; por lo que se acepta que existen puntuaciones altas en el pensamiento crítico que están asociadas a las puntuaciones altas en el valor de liderazgo, aceptándose la hipótesis alterna como respuesta a este análisis: A mayor valor interpersonal de liderazgo mayor pensamiento crítico.

\section{Discusión}

Teniendo en cuenta que el pensamiento crítico es la disposición que adopta el sujeto al analizar, interpretar y evaluar la información que dispone (Zaldivar, 2010) esta ha de ser trabajada en todo el proceso de enseñanzaaprendizaje. En el presente estudio según los resultados obtenidos, se señala que la percepción del pensamiento crítico en las estudiantes ingresantes del I ciclo se encuentra en un 53,9\% en el nivel promedio y en un $40 \%$ en el nivel alto, en una relación tendente a asumir la realidad, interpretarla y dar juicios de valor coherente con el entorno. 
En cuanto a los valores interpersonales los estudiantes demuestran una interiorización de ellas en $67 \%$ en el nivel alto la cual indica que el tema axiológico es estimado por los estudiantes como un factor importante de las relaciones humanas.

Por otra parte, el estudiante del siglo XXI en la construcción de sus conocimientos ha de partir de una profunda base axiológica que le incentive a ser cada día mejor en todos los ámbitos de su vida como también en la capacidad de analizar el entorno, evaluar la realidad y dar un juicio de razón sustentada, en otros términos, el pensar críticamente. En nuestro estudio al correlacionar el pensamiento crítico con los valores interpersonales se obtuvo un p-valor de 0,513 señalando una correlación positiva de baja intensidad. Esto nos ha de llevar a reflexionar que al centrarnos en los estudiantes universitarios como futuros entes sociales que contribuirán al desarrollo del país con su trabajo y participación en la vida política de la nación es necesario que se afiance en ellos cualidades no solo intelectuales sino también valorativas de manera vivencial y por lo menos así asegurarnos personas comprometidas con la verdad, honestidad de acciones y no sucumban ante la corrupción. (Deroncele-Acosta., Nagamine-Miyashiro y Medina-Coronado, 2020).

La base del desarrollo de esta competencia del pensamiento crítico se encuentra en los estudios de educación básica, que lamentablemente en algunas instituciones educativas no lo trabajan con la seriedad debida o no le dan mucha importancia, pues prefieren poner todo su esfuerzo en las áreas de comunicación y matemáticas buscando mecánicamente las respuestas inmediatas y no el análisis juicioso. Entonces el estudiante al llegar a los primeros ciclos de los estudios de pregrado encuentra dificultad en el proceso de trabajo investigativo y de pensar críticamente la realidad. A parte de ellos se ha de considerar que el desarrollo del pensamiento crítico es también un trabajo que involucra al docente universitario que son los encargados de configurar el perfil del egresado a través de las prácticas educativas. (Cangalaya, 2020).

El ejercicio de pensar de manera "juiciosa o reflexiva" (Facione, 1990 p. 15) es decir crítica, implica ser intelectualmente disciplinado con capacidad de evaluar la realidad en la diversidad de sus factores dando también un valor explicativo a las creencias y motivaciones subyacentes en el sujeto que vive la experiencia.

Un estudio interesante, es el que realizó Castro y González-Palta (2016) con estudiantes de la carrera de Psicología donde se favoreció el uso de grupos de Facebook junto con una metodología aplicada para el desarrollo del Pensamiento crítico. Es así como la percepción de los estudiantes en la participación de la página de Facebook creada con el nombre de "Face-Critic", afirman que hay un desarrollo del pensamiento crítico que vio reflejado en un $88 \%$ en este rubro y que el espacio paralelo creado para pensar en los temas de personalidad llegó a un al $72 \%$ de aceptación producto de la interacción con esta red social. Este espíritu crítico no quedará circunscrito dentro del aula, sino que se traducirá luego en la curiosidad intelectual por buscar, contrastar, sistematizar, verificar y comunicar la información recibida que también resulta ser característica del pensamiento científico. Entonces: "Para que el alumno aprenda un concepto es necesario primero internalizarlo, para poder después aplicarlo y observar el valor". (Núñez-López., Ávila-Palet y Olivares-Olivares, 2017, p.86). 
Los resultados revelan, entonces que hay que afianzar las prácticas para el ejercicio del pensamiento crítico en los estudiantes de manera reiterativa, siendo ésta un trabajo conjunto de la plana docente y de los estudiantes donde el hombre y al mujer tienen la misma posibilidad y no hay diferencia por sexo como lo señala los hallazgos de Salazar-Blandón y Ospina-Rave (2019) donde el nivel promedio de Pensamiento Crítico para hombres fue de $50,45 \pm 13,20$, y para mujeres de $48,04 \pm 9,79$ $(\mathrm{t}=0,946, \mathrm{p}=0,346)$. De igual forma en el trabajo de Salahshoor y Rafiee (2016) que en una muestra en institutos de idioma privado no se encontró diferencias hombres $(43,2$ $\pm 9,07)$ y mujeres $(40,94 \pm 7,37 \mathrm{p}=0,064)$.

Sin duda, en estos últimos tiempos ha tomado importancia los trabajos orientados a la investigación científica como una competencia estratégica. Las universidades son espacios que se promueven el análisis científico de la realidad, la argumentación, la comprensión, la verificabilidad de los hechos, la solución de problemas, etc. que resulta ser características también parte del pensamiento crítico. Es así que se tiene que los resultados de Lévano, (2020) con estudiantes de la carrera de Traducción demostró la influencia de su programa en el desarrollo de pensamiento crítico en la adquisición de competencias estratégicas a través de actividades cognitivas y metacognitivas. El porcentaje alcanzado en el rubro de Capacidad de resolver problemas del grupo experimental alcanzo una diferencia de $30 \%$ en la identificación de problemas, un 27 $\%$ en la selección de estrategias con un $40 \%$ de diferencia frente al grupo control en la descripción de problema.

Es cierto también, que existen estudiantes de pregrado que no logran filtrar con claridad los datos de la realidad socio cultural, de apreciar cuáles son las fuentes de información primarias de la literatura "gris", que dato encontrado no está basada de veracidad o se presenta como pseudo ciencia, etc. Pero esta capacidad de distinción se alcanza también con el trabajo mancomunado e interpersonal, del compartir experiencias, emociones, valores y debatirlas con otros compañeros. El pensamiento crítico como una habilidad de orden superior resulta ser una herramienta que no solamente de uso académico sino participativo en vista del bien común y formativo para toda la vida, siendo inclusive más importante que las calificaciones o estar en el tercio superior del ciclo de estudio. De ahí la importancia, que consideremos el pensamiento crítico que ayuda al "desarrollo de las habilidades para cooperar con los miembros del equipo en aras de alcanzar un objetivo común" (LatorreCosculluela; et. al. 2020 p.8)

Finalmente, se ha de promover en los estudiantes el desarrollo de la experiencia valorativa desde el primer ciclo de estudio que fomente el pensar crítico y creativo, usando los medios físicos como virtuales. En ella, no solo están los conocimientos intelectuales, sino también, los afectivos que impulsaran al estudiante contrastar la realidad de manera comprometida, asertiva y empática, más allá del pensamiento tradicional de corte lineal y deductivo.

\section{CONCLUSIONES}

El ser formado en valores o asumir estos como estándares de comportamiento ayudará a ver entre la niebla el mejor camino a seguir, puesto que la reflexión valorativa implica el ejecutar habilidades cognitivas elevadas que son también capacidades del pensamiento crítico. 
El buscar un método único para la "aprehensión" de valores a manera de una guía estandarizada no resulta viable, en cuanto lo diversos que somos por tradiciones familiares, experiencias vividas, lugares académicos de formación y por ambiente cultural donde provenimos. Lo que si es cierto es que se ha de formar el horizonte valorativo en la cual todos deseemos estar, que entendamos que el valor es un medio para alcanzar cosas mejores respetando nuestro al prójimo y a nuestro medio ambiente.

El desarrollo del pensamiento crítico en su construcción implica una serie de acciones que ponga en juego y relevancia dicha competencia. Encausarla dentro de las actividades del aprendizaje significativo y participativo, favoreciendo de manera especial el trabajo con los estudiantes de pregrado donde el docente puede programar una serie de acciones para el ejercicio de esta competencia especifica dentro de cualquiera de las asignaturas de su carrera profesional. En esta perspectiva, el pensamiento crítico será llave maestra que abra el gusto y aprecio, entre otras cosas, por una experiencia valorativa que comenzó en casa, se afianza en la vida universitaria y que se mantendrá a lo largo de nuestra vida.

\section{REFERENCIAS}

Cangalaya, L. (2020). Habilidades del pensamiento crítico en estudiantes universitarios a través de la investigación. Desde el Sur, 12(1), pp. 141-153

Casares, D., González, J. y Siliceo, A. (1999). Liderazgo, valores y cultura organizacional. McGraw-Hill

Castro, P. y González-Palta, I. (2016). Percepción de Estudiantes de Psicología sobre el uso de Facebook para Desarrollar Pensamiento Crítico. Formación universitaria, $9(1)$,
pp.4556https://dx.doi.org/10.4067/S071 8-50062016000100006

Deroncele-Acosta, A., Nagamine-Miyashiro, M., y Medina-Coronado, D. (2020). Desarrollo del pensamiento crítico. Maestro Y Sociedad, 17(3), 532-546. Recuperado de https://maestroysociedad.uo.edu.cu/inde x.php/MyS/article/view/5220

Dicado, M.A; Campi C.F y Saa, A. S. (2019). Los Valores Humanos Y Su Incidencia en La Calidad Del Estudiante Universitario. Opuntia Brava, 11(3), 247-255. Recuperado de: https://doi.org/10.35195/ob.v11i3.809

Facione, P. (2007). Pensamiento crítico: ¿Por qué es importante? Recuperado de: http://eduteka.icesi.edu.co/pdfdir/Pensa mientoCriticoFacione.pdf

Facione, P. (1990), "Critical thinking: a statement of expert consensus for purposes of educational assessment and instruction", en American Philosophical Association, California, The California Academic Press, pp. 1-111

Frondizi, R. (1972). ¿Qué son los valores? Fondo de Cultura Económica

Gázquez-Linares, J. J., Pérez-Fuentes, M. C., Carrión-Martínez, J. J., de la Rosa, A. L., y Molero, M. M. (2015). Interpersonal value profiles and analysis to adolescent behavior and social attitudes. [Perfiles de valores interpersonales y análisis de conductas y actitudes sociales de adolescentes] Revista De Psicodidactica, 20(2), 321-337. doi:10.1387/RevPsicodidact.12978

Gordon L. (1977). Cuestionario de Valores Interpersonales (SIV).TEA

Kleptsova, E. Y., Kleptsov, N. N., Mishutinskaya, E. A., Shubnitsyna, T. V., y Tsvetkova, N. V.(2018). Psychological structure of humane interpersonal relations among the subjects of educational activity. Espacios, 39(49). Recuperado de: http://www.revistaespacios.com/a18v39 
n49/a18v39n49p02.pdf

Latorre-Cosculluela, C; Vazquez-Toledo, S; Rodriguez-Martinez, A y Liesa-Orus, M. Design Thinking: creatividad $\mathrm{y}$ pensamiento crítico en la universidad. REDIE[online]. 2020, vol.22, e28. Epub 02Mar-2021. ISSN 1607-4041. https://doi.org/10.24320/redie.2020.22. e28.2917

Lévano, S. (2020). Pensamiento crítico y adquisición de la competencia estratégica en estudiantes de traducción. Educación, 29(56), 73-94. https://dx.doi.org/10.18800/educacion.2 02001.004

Núñez-López, S., Ávila-Palet, J. y OlivaresOlivares, S. (2017). El desarrollo del pensamiento crítico en estudiantes universitarios por medio del Aprendizaje Basado en Problemas. Revista iberoamericana de educación superior, 8(23), 84-103. http://www.scielo.org.mx/scielo.php?scri pt=sci_arttextypid=S200728722017000300084ylng=esytlng=es

Pérez, M. Linares, J. J. G, Molero, M, Soler, F. J., y Martín, A. B. B. (2015). Interpersonal values related to aggressor and victim profiles in adolescents. [Valores interpersonales relacionados con el perfil de agresor y víctima en adolescentes] International Journal of Psychology and Psychological Therapy, 15(2), 229-239.
Recuperado de: https://www.ijpsy.com/volumen15/num 2/416/valores-interpersonalesrelacionados-con-ES.pdf

Richard, P. y Elder, L. (2005), Una guía para los Educadores en los Estándares de Competencia para el Pensamiento Crítico. Estándares, Principios, Desempeño. Indicadores y Resultados con una Rúbrica Maestra en el Pensamiento Crítico. Recuperado de: https://www.criticalthinking.org/resourc es/PDF/SP-Comp_Standards.pdf

Rokeach, M. (1973): The nature of human values. N.York: Free Press

Salahshoor N, Rafiee M. (2016). The relationship between Critical Thinking and gender: A case of Iranian EFL learners. J Appl Linguist Lang Res [Internet]. 2016; 3(2):117-23. Available from:

http://www.jallr.com/index.php/JALLR/a rticle/viewFile/219/ pdf219

Watson, G. y Glaser, E. (1980). Critical Thinking Appraisal. San Antonio: Harcourt Brace Jovanovich

Zaldívar, P. (2010). El constructo pensamiento crítico. Recuperado de: https://www.yumpu.com/es/document/r ead/13031876/2010-elconstructopensamiento-criticouniversidad-de-zaragoza 